\title{
Improvement of back-azimuth estimation in real-time by using a single station record
}

\author{
Shunta Noda, Shunroku Yamamoto, Shinji Sato, Naoyasu Iwata, Masahiro Korenaga, and Kimitoshi Ashiya
}

Railway Technical Research Institute, 2-8-38 Hikari-cho, Kokubunji-shi, Tokyo 185-8540, Japan

(Received August 4, 2011; Revised October 1, 2011; Accepted October 18, 2011; Online published March 12, 2012)

\begin{abstract}
We propose a new approach to improve the accuracy of the back-azimuth estimation in real-time by using a single station record. Compared with the conventional approach in which the length of the time window for the analysis is fixed for all data, the accuracy and speed of the estimation are drastically improved by introducing a variable-length time window which is determined by the first half cycle of the wavelength of the initial $P$-wave, because this window tends to reduce the influences of trailing scattered waves. The analysis, using the K-NET dataset, shows that the estimation, using this new approach, is improved by $28 \%$ and $0.25 \mathrm{~s}$ in accuracy and speed, respectively.
\end{abstract}

Key words: Earthquake early warning, back-azimuth, principal component analysis, single station method, onsite method.

\section{Introduction}

The Earthquake Early Warning (EEW) system is a very effective approach to reducing earthquake hazards. In general, the EEW system can provide earthquake information, e.g. the magnitude and hypocenter, or epicenter, location, within several seconds after a $P$-wave is detected at the first station. In Japan, practical EEW systems have been developed for approximately 20 years. Nakamura (1988) developed the Urgent Earthquake Detection and Alarm System (UrEDAS) in order to halt the Shinkansen (bullet train) safely during earthquakes. In the UrEDAS, the magnitude is estimated from the predominant frequency of the $P$-wave for the initial 3 seconds, and then the epicentral distance is estimated from the magnitude and amplitude by using a predefined attenuation relation. This idea is adopted by Allen and Kanamori (2003) and Wu and Kanamori (2005). It is called the $\tau_{\mathrm{c}}$ method.

The Japan Meteorological Agency (JMA) collaborated with the Railway Technical Research Institute (RTRI) and the National Research Institute for Earth Science and Disaster Prevention (NIED) in developing an EEW system, and then started to give alarm information to the public in 2007 (Horiuchi et al., 2005; Hoshiba et al., 2008; Nakamura et al., 2009). In this system, hypocenter locations are determined by a combination of several techniques. In the case that an earthquake is detected at only a single station, epicenter locations are determined by a combination of the following two kinds of methods (known as the single station method, or the on-site method). One is the Principal Component Analysis (PCA) (Meteorological Research Institute, 1985) (Fig. 1) by which the back-azimuth is estimated from

Copyright (c) The Society of Geomagnetism and Earth, Planetary and Space Sciences (SGEPSS); The Seismological Society of Japan; The Volcanological Society of Japan; The Geodetic Society of Japan; The Japanese Society for Planetary Sciences; TERRAPUB.

doi:10.5047/eps.2011.10.005 the 1st principal component of the particle motion of the initial $P$-wave. The 1 st principal component is the direction with the minimum variance for the points of observed displacement. The other method is the $B-\Delta$ method (Odaka et al., 2003) by which the epicentral distance is estimated from a coefficient $B$ of a fitting function $B t * \exp (-A t)$. The characteristic of this method is to determine the epicentral distance at first, and then the magnitude is estimated from the epicentral distance and amplitude by using a predefined attenuation relation. Although the estimation order is the reverse, compared with the method in the UrEDAS, we consider that the method, by which the epicentral distance is estimated at first, would be more reliable for a large earthquake, because it can continue determining the magnitude during the spreading of the seismic rupture.

In the JMA system, when the number of stations which detect the earthquake is increased, the so-called Territory method, the Grid search method and the $T_{\text {now }}$ method (Horiuchi et al., 2005), are used to determine hypocenter locations (this is called the multi-station method or network method). The magnitude is calculated from the hypocenter location and amplitude by using a pre-defined attenuation relation.

RTRI developed a new EEW system for Shinkansen and replaced the UrEDAS with the new one in 2004-2005 (Ashiya, 2002; Iwahashi et al., 2004; Yamamoto et al., 2011). In this system, the PCA and the $B-\Delta$ method are also used to determine epicenter locations.

In general, earthquake information estimated from the single station method is obtained more quickly, and, yet, with a lower accuracy compared with that estimated from the multi-station method. Therefore, an improvement in accuracy using the single station method is essential in order to make the present EEW systems more stable. The speed should also be improved so as to make the present systems more effective. 


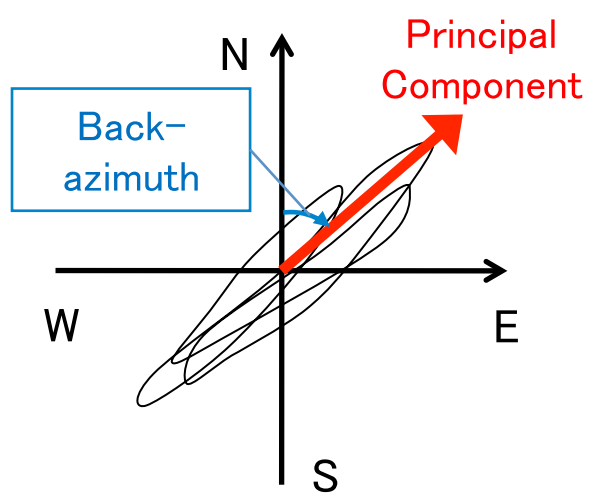

Fig. 1. Estimation of the back-azimuth by using the Principal Component Analysis (PCA). The principal component equals the direction in which the sum of the distances between the principal component and points (amplitude of the particle motion of every sample) is minimized.

In this study, we propose a new approach to improve the accuracy of the PCA, which estimates the back-azimuth by introducing a variable-length time window instead of the conventional fixed-length time window.

\section{Conventional Approach}

In this section, we present the conventional approach to estimate the back-azimuth by using the single station method, and we evaluate its accuracy by analyzing the KNET data.

First, the analytical steps of the conventional approach are shown as follows:

(1) The arrival time of the $P$-wave is calculated using the STA/LTA (Short Time Average to Long Time Average) method (e.g. Horiuchi et al., 2005).

(2) The displacement data is calculated from the observed acceleration records by using the IIR (Infinite-duration Impulse Response)-filter.

(3) The displacement data is band-passed for 1-2 Hz by using the IIR-filter.

(4) The back-azimuth is determined from the 1st principal component of the particle motion of the initial $P$-wave by using the PCA (Fig. 1). The length of the time window for the analysis is set to be $1.1 \mathrm{~s}$ (start time is the $P$-wave arrival). The important point is that the length of the time window is fixed for all data.

$1.1 \mathrm{~s}$ was selected as the length of the time window in the conventional approach by using the limited amount of the dataset, when the conventional approach was developed. However, we consider that a more detailed analysis was necessary for a more reliable approach with an updated dataset.

Next, we evaluate the accuracy of the back-azimuth estimated from the conventional approach. Waveform data observed at K-NET stations operated by NIED are used for the evaluation. The number of the waveform data is 1,991 , which were selected with the following requirements; the period ranges from 1995 to 2010 , the magnitudes exceed 5.5 , the epicentral distances range within $300 \mathrm{~km}$, the JMA seismic intensities exceed 3.5.

Figure 2 shows the frequency of errors of the back-

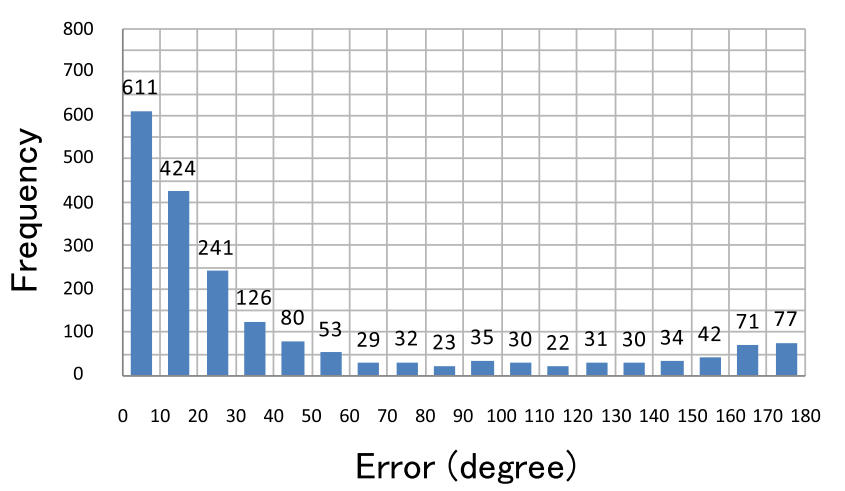

Fig. 2. Frequency of the errors of the back-azimuth estimated from the conventional approach by using a K-NET dataset.

azimuth estimated using the conventional approach from the dataset as described above. In this case, the error means the absolute value of the difference between the estimated back-azimuth and the value determined by JMA published data. As a result, the errors of approximately $64 \%$ of the dataset are ranged within 30 degrees, and the rms (root mean square) of the errors is 67.9 degrees. Obviously, data whose error range lies between 160 and 180 degrees (this means that the estimated back-azimuths turn almost in a reverse direction from the true ones) are relatively frequent compared with data whose error range lies between 60 and 160 degrees.

\section{Relationship between the Fixed Time Window and Errors}

In this section, we evaluate the accuracies of the errors in the case where the length of the fixed time windows are varied from 0.1 to $2.0 \mathrm{~s}$ by $0.1 \mathrm{~s}$. The dataset used is the same as the one used in Section 2.

The rms of the errors obtained by the analysis is shown in Fig. 3. When the length of fixed time window is set to be $0.6 \mathrm{~s}$, the rms is found to be a minimum ( 57.3 degrees). This result indicates that the fixed 0.6-s time window improves by approximately $16 \%$ in accuracy compared with the conventional time window $(1.1 \mathrm{~s})$. The rms of the errors increases with a longer time window, excluding an extremely short time window (from 0.1 to $0.3 \mathrm{~s}$ ) (Fig. 3). We consider that this is because the longer time windows are more largely contaminated by trailing scattered waves than the direct $P$-wave.

Figure 4 shows the frequency of the errors of the backazimuth estimated from the fixed 0.6-s time window. From this figure, it is found that the data, whose error range is within 60 degrees, are increased, and the other data are decreased compared with the result by using the conventional time window (Fig. 2). The data, whose error range is between 160 and 180 degrees, are not relatively frequent compared with data whose error range is between 60 and 160 degrees. This result suggests that mistakes concerning the polarity of the initial $P$-waves are often made when the conventional time window is used. 


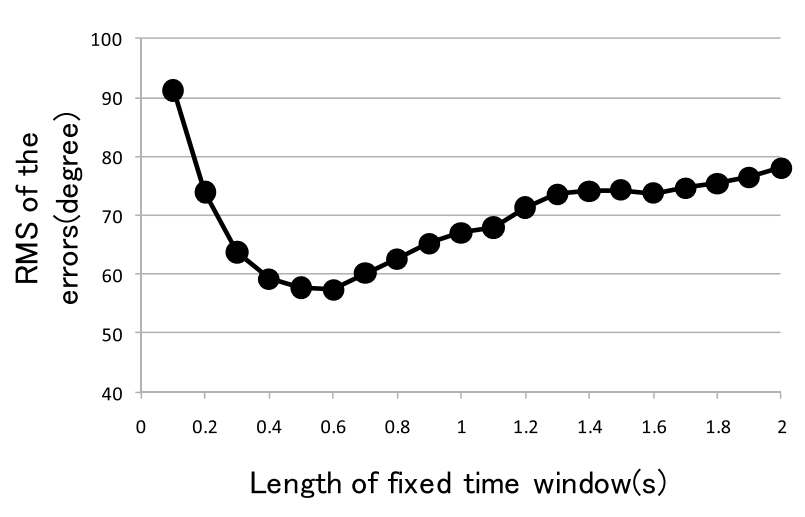

Fig. 3. Rms of the errors in the case that the fixed time window lengths are varied from 0.1 to $2.0 \mathrm{~s}$ in steps of $0.1 \mathrm{~s}$.

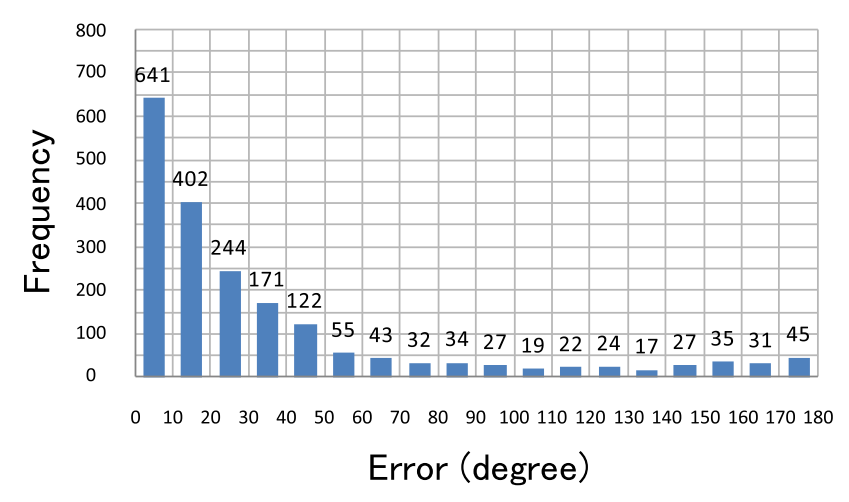

Fig. 4. Frequency of the errors of the back-azimuth estimated from the fixed $0.6 \mathrm{~s}$ time window.

\section{Variable Time Window}

In order to reduce the influences of the trailing scattered waves more effectively, we propose a variable time window approach to calculate the back-azimuth of a pure $P$-wave. In this case, the variable time window means the length of the first half cycle of the wavelength of the initial $P$-wave in the band-pass displacement data (Fig. 5). In detail, its length equals the time from the $P$-wave arrival to the first zero-cross point. The important point of this new approach is that the lengths of the time window vary according to the characteristics of each datum.

Figure 6 shows the frequency of the lengths of the variable time window for the dataset described above. From this figure, it is found that data around $0.4-0.6 \mathrm{~s}$ is the most frequent. The average of the lengths is $0.85 \mathrm{~s}$. It is important that this average is shorter than the length of the conventional time window.

Figure 7 shows the frequency of the errors by using a variable time window. If the length of the variable time window is less than $0.2 \mathrm{~s}(1.8 \%$ of the data) or cannot be determined within $2 \mathrm{~s}$ ( $4.5 \%$ of the data), $0.6 \mathrm{~s}$ is used as the length of the time window. Consequently, data, whose error range is within 30 degrees, are increased and the other data are decreased by comparing Fig. 4 (fixed $0.6 \mathrm{~s}$ time window) with Fig. 7 (variable time window). The rms of the errors is 49.0 degrees when the variable time window is used. This result demonstrates that the variable time window results in an improvement of approximately $28 \%$, or

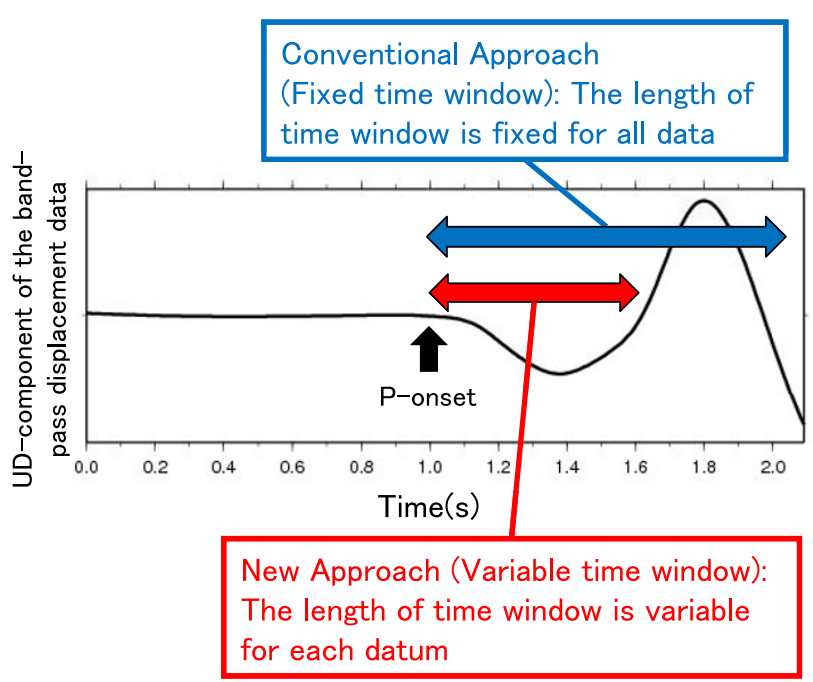

Fig. 5. Comparison between the conventional fixed time window and the new variable time window. In the new approach, the length from a $P$-wave arrival to the first zero-cross point (first half cycle of the wavelength of the initial $P$-wave) of the UD component of the band-pass displacement data is adopted as the time window.

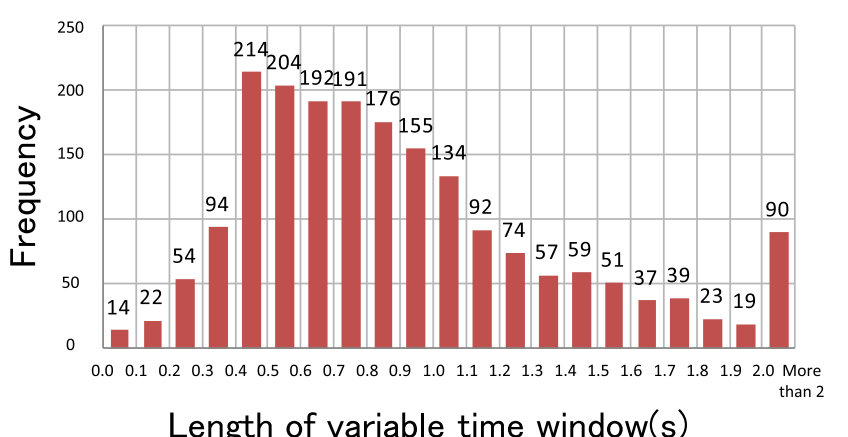

Fig. 6. Frequency of the length of the variable time window.

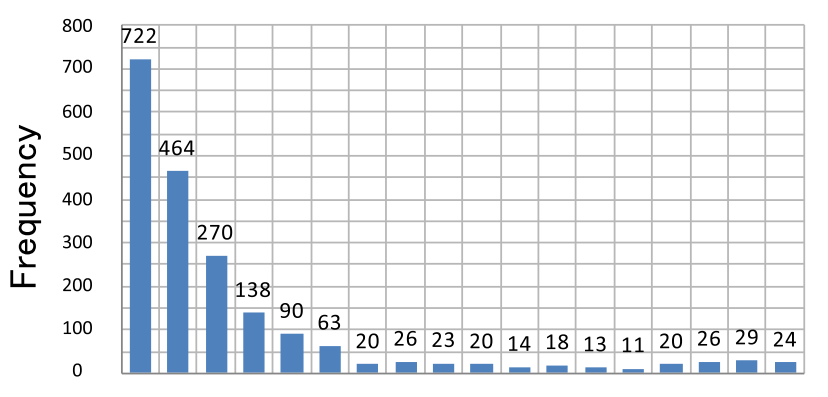

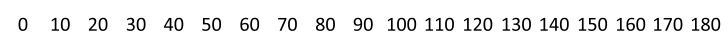

Error (degree)

Fig. 7. Frequency of the errors of the back-azimuth estimated from the variable time window.

$14 \%$, in accuracy compared with the result obtained using the conventional approach, or that by using a fixed 0.6-s time window, which is the most accurate when a fixed time window is used, respectively. 

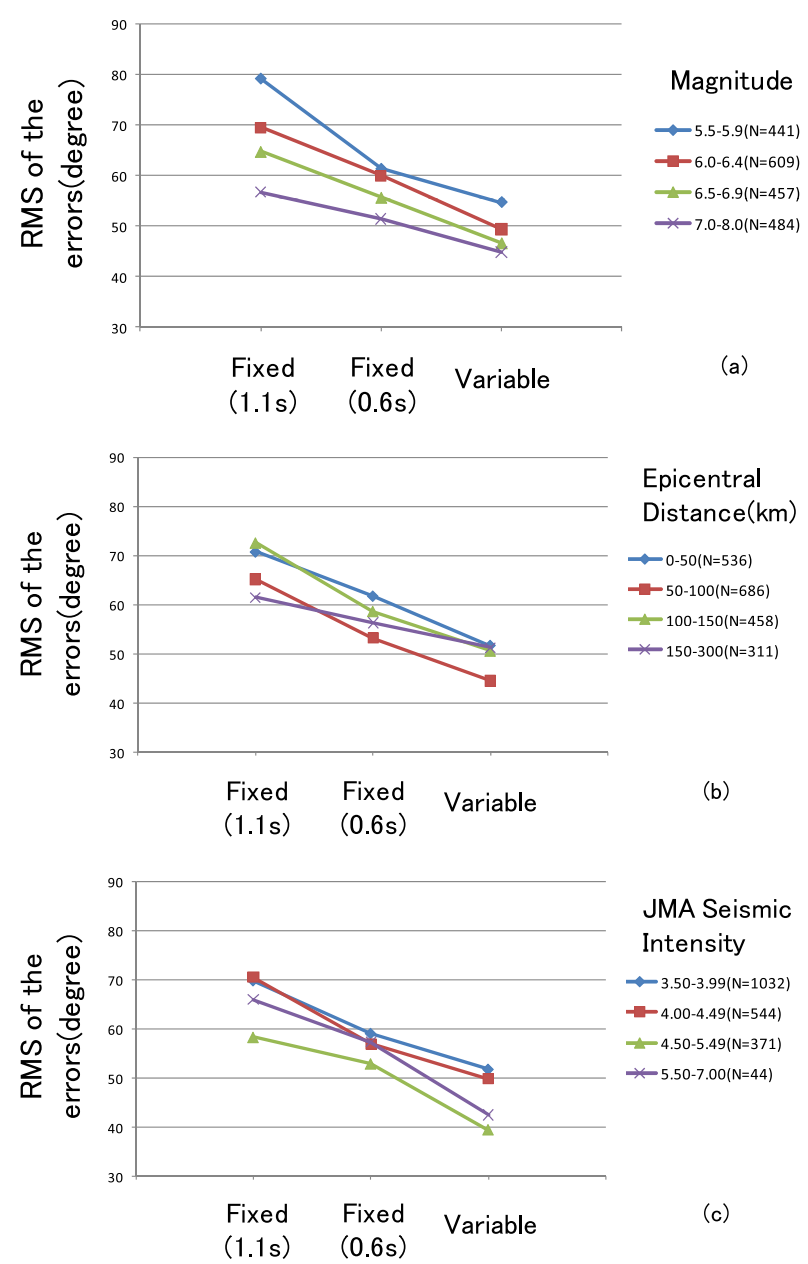

Fig. 8. Rms of the errors when two fixed time windows (1.1 s, $0.6 \mathrm{~s})$ and a variable time window are used. The results shown in (a), (b), and (c) are for magnitude, epicentral distance and JMA seismic intensity, respectively.

\section{Discussion}

We have investigated difference in the improvements in accuracy for each parameter (magnitude, epicentral distance, and JMA seismic intensity) when two fixed time windows $(1.1 \mathrm{~s}, 0.6 \mathrm{~s})$, and a variable time window, are used (Fig. 8). From Fig. 8(a), it is found that the shorter time window is appropriate even in the case of a large earthquake, compared with the result by using a fixed 1.1-s time window with that using a fixed 0.6-s time window. Consequently, the rms of the errors, from using a variable time window, is the lowest in the case of every parameter. This fact indicates that the new approach is so robust that the accuracy of the back-azimuth, estimated from the PCA, is statistically improved for every parameter.

As mentioned above, lengths in the range 0.4-0.6 s are the most frequently occurring in the appraisal of the length of the variable time window (Fig. 6). In addition, in the cases that fixed time windows of various lengths are used, rms of the errors is almost a minimum when the length of the fixed time window is in the range $0.4-0.6 \mathrm{~s}$ (Fig. 3). We consider that this coincidence shows the appropriateness of the above analyses.

The effect of the new approach not only improves the ac- curacy of the back-azimuth, but also reduces the time for the estimation compared with the conventional approach, because the average of the length of the variable time window is shorter than that of the conventional time window, as described above.

Furthermore, the new approach has two practical advantages for the present EEW systems. The first is that the new approach is so simple that the CPU requirement will hardly be increased even in a real-time process. The second is that the new approach needs only the addition of several code lines to calculate the length for the first half cycle of wavelength of an initial $P$-wave to the source code of the present systems.

\section{Conclusion}

We have proposed a new approach for improving the accuracy and speed of back-azimuth estimation from the PCA by introducing a variable time window to reduce the influences of trailing scattered waves, instead of a conventional fixed time window. By using the new approach, it is found that the accuracy and speed of the estimation is improved by $28 \%$ and $0.25 \mathrm{~s}$, respectively, compared with the conventional approach. The effect of the new approach is so robust that the accuracy is statistically improved independent of magnitude, epicentral distance and JMA seismic intensity.

Acknowledgments. We would like to thank the National Research Institute for Earth Science and Disaster Prevention for enabling us to use the waveform data observed at K-NET. We also wish to thank Dr. Mitsuyuki Hoshiba and an anonymous reviewer for their helpful comments.

\section{References}

Allen, R. M. and H. Kanamori, The potential for earthquake early warning in Southern California, Science, 300, 786-789, 2003.

Ashiya, K., Development of a new early earthquake detection and alarm system, Quart. Rep. RTRI, 43(2), 50-52, 2002.

Horiuchi, S., H. Negishi, K. Abe, A. Kamimura, and Y. Fujiwara, An automatic processing system for broadcasting earthquake alarms, Bull. Seismol. Soc. Am., 95(2), 708-718, 2005.

Hoshiba, M., O. Kamigaichi, M. Saito, S. Tsukada, and N. Hamada, Earthquake early warning starts nationwide in Japan, Eos Trans, $A G U, \mathbf{8 9}$, 73-74, 2008.

Iwahashi, H., N. Iwata, S. Sato, and K. Ashiya, Practical use of earthquake quick alarm system, RTRI Report, 18(9), 23-28, 2004 (in Japanese).

Meteorological Research Institute, Study on earthquake prediction by geophysical method, Technical Reports of the Meteorological Research Institute, No. 16, 1985 (in Japanese).

Nakamura, H., S. Horiuchi, C. Wu, S. Yamamoto, and P. A. Rydelek, Evaluation of the real-time earthquake information system in Japan, Geophys. Res. Lett., 36, L00B01, doi:10.1029/2008GL036470, 2009.

Nakamura, Y., On the urgent earthquake detection and alarm system (UrEDAS), Proceedings of Ninth World Conference on Earthquake Engineering, VII, 673-678, 1988.

Odaka, T., K. Ashiya, S. Tsukada, S. Sato, K. Ohtake, and D. Nozaka, A new method of quickly estimating epicentral distance and magnitude from a single station record, Bull. Seismol. Soc. Am., 93(1), 526-532, 2003.

$\mathrm{Wu}$, Y. M. and H. Kanamori, Experiment on an onsite early warning method for the Taiwan early warning system, Bull. Seismol. Soc. Am., 95(1), 347-353, 2005.

Yamamoto, S., S. Sato, N. Iwata, M. Korenaga, Y. Ito, and S. Noda, Improvement of seismic parameter estimation for the Earthquake Early Warning System, Quart. Rep. RTRI, 52(4), 206-209, 2011.

S. Noda (e-mail: noda@rtri.or.jp), S. Yamamoto, S. Sato, N. Iwata, M. Korenaga, and K. Ashiya 